\title{
PRELIMINARY ASSESSMENT OF ALLELOPATHC EFFECTS OF ROOT AND PLANT RESIDUE LEACHATES ON ROTATIONAL CROPS AND RYEGRASS
}

\author{
Michael Ignatius Ferreira ${ }^{1^{*}}$, Carl Frederick Reinhardt ${ }^{2}$ and Marieta van der Rijst ${ }^{3}$ \\ ${ }^{1}$ Directorate of Plant Sciences, Western Cape Department of Agriculture, Private Bag X1, Elsenburg, 7607 Republic \\ of South Africa \\ ${ }^{2}$ Unit for Environmental Sciences and Management, School of Geo- and Spatial Sciences, North-West University, \\ Potchefstroom, 2531 Republic of South Africa \\ ${ }^{3}$ Agricultural Research Council: Biometry Unit, Private Bag X5013, Stellenbosch, 7599 Republic of South Africa
}

https://doi.org/10.35410/IJAEB.2021.5673

\begin{abstract}
Allelopathy is an important mechanism of interference, affecting crop performance. In this preliminary study, the aim was the assessment of allelopathic effects on plant growth and biomass production in a glasshouse. For this evaluation, pot leachates obtained from roots and plant residues from rotational crops and ryegrass was utilised to determine mutual allelopathic interactions. Experiment I was set up to determine effects from root leachates from pots planted to each plant species. For Experiment II, aboveground plant material collected separately, airdried and ground to a coarse powder was utilised to prepare aqueous infusions for testing. At both three and five weeks, root leachates from alfalfa $(-18 \%$ and $-17 \%)$ and medic $(-10 \%$ and $9 \%$ ) caused a reduction in barley plant height. Wheat dry mass production was reduced by aboveground leachates from barley $(-32 \%)$, canola $(-52 \%)$, alfalfa $(-76 \%)$ and ryegrass $(-54 \%)$. Alfalfa root leachates caused a reduction in ryegrass plant height $(-16 \%)$ at three weeks. The current preliminary assessment indicated that lupine, alfalfa and medic contain possible allelochemicals that might be responsible for the inhibition or stimulation exhibited on the growth and biomass mass production of the selected plant series. Future research should elucidate the specific allelopathic compounds in these crop plants and its use as mulches that suppress weeds.
\end{abstract}

Keywords: leguminous crops, inhibition; stimulation; root and aboveground leachates, weed suppression.

\section{INTRODUCTION}

Some plants affect the development of neighbouring plants by releasing secondary metabolites into their environment. This phenomenon is known as allelopathy and is a potential tool for weed management within the framework of sustainable agriculture (Lebecque et al., 2018). Allelopathy encompasses complex effects of one plant on another that may be modified by leachates from roots or plant litter (Pelissier, 2013). Agriculturalists and ecologists have debated the evolutionary origin and competitive advantage of this phenomenon for a long time, but over the last several years, allelopathy has been widely accepted as a mechanism underlying the invasion success of weeds (Li et al., 2015; Christina et al., 2015). Research by Bais et al. (2003) suggested that allelopathic properties could render one species more invasive to native species and thus detrimental to an agricultural setting. This corroborated findings by Weston (2005) who 
Vol. 06, No. 05; 2021

ISSN: $2456-8643$

reported that alterations in the environment by various plant interference mechanisms can differentially affect neighbouring plant species. More specifically, Belz (2004) suggested that crop allelopathy can be exploited for weed management through the release of allelochemicals from intact roots of living plants or decomposition of plant residues. Allelochemicals are released into the environment directly by live plants as volatiles, leachates and/or root exudates (De Gouveia Baratelli et al., 2012).

According to Brussaard et al. (2007) sustainable crop productivity is dependent on the ability to maintain ecological functions supported by soil biota. Foley et al. (2011) emphasized that modern agriculture calls for sustainable systems that can maintain productivity while reducing inputs and production losses and increasing the biodiversity of agro-ecosystems. Furthermore, Foley et al. (2011) speculated that understanding the role of root exudates in plant recognition and competition is likely to have significant impacts on agricultural productivity.

Canarini et al. (2019) confirmed that plant community composition responds to environmental stresses and to competition for available resources, either aboveground (e.g., light) or belowground (e.g., nutrients and water). In addition, the majority of root exudates, including primary metabolites (sugars, amino acids, and organic acids) are lost passively from the root and used by rhizosphere-dwelling microbes (Canarini et al., 2019). Thus, the fate and effectiveness of allelopathic compounds in soil are highly dependent on biotic and abiotic environmental conditions (Lorenzo et al., 2013).

Allelochemicals released from plants are produced as byproducts of plant metabolic pathways (Makoi and Ndakidemi, 2012). Plants, for their part, exude a range of carbon metabolites that can act as a food and energy source for use by microbes (Kuzyakov and Blagodatskaya, 2015). Root exudates include primary metabolites (sugars, amino acids, and organic acids) and determine plant interactions with the soil environment (Canarini et al., 2019). The process of allelopathy is set in motion when plants release allelochemical compounds into the environment by different ways such as leaching from the plant shoots by rainfall, releasing volatile phytotoxic compounds from a plant's green parts, releasing phytotoxic compounds from decomposed plant material and eventually, releasing phytotoxic compounds by the root exudates (Amini et al., 2012).

Nevertheless, interference mechanisms by weeds with crops encompass both competition for growth factors and the release of chemical compounds. In agricultural ecosystems, the latter is an important mechanism of interference, affecting crop performance (Batish et al., 2002). Contrary to earlier reports that it only leads to growth suppression, allelopathy is a chemical-mediated process which may be stimulatory or inhibitory (Belz et al., 2005).

For the detection and evidence of allelopathic effects, it has to be released into soils by leachates and persist at phytotoxic levels in the field to influence a target plant (Li et al., 2015). Where allelopathic weeds are involved, it can affect crops in a number of ways, like delaying or preventing seed germination and reducing seedling growth (Bouhaouel et al., 2016). Furthermore, Li et al. (2015) reported that factors influencing these processes include soil texture, chemical composition and the community of microorganisms. 
Vol. 06, No. 05; 2021

ISSN: $2456-8643$

One approach suggested by Kumar et al. (2009) to understand the allelopathic effects is to determine which parts of a plant's root or shoot, has the most suppressive effects on another plant's emergence and growth. In this regard, Das \& Bandyopadhyay (2011) showed that aqueous extracts of some plants inhibit root and shoot growth. However, for most plant species, shoot extracts were more effective than root extracts in inhibiting seed germination and growth of downy brome. Results by Kumar et al. (2009) showed that shoot extracts of two goldenrod species (Euthamia graminifolia L. Nutt. and Solidago canadensis L.) had inhibitory effects on both germination and growth of radish (Raphanus sativus L.) and lettuce (Lactuca sativa L.). By contrast, root extracts had no inhibitory effects on germination of these two species, but suppressed root growth. Aqueous shoot extracts of buckwheat stimulated Powell amaranth (Amaranthus powellii S. Wats.) germination slightly, but inhibited radicle growth (Kumar et al., 2009). Aqueous soil extracts from buckwheat-amended soil, inhibited germination of Powell amaranth.

Several reports confirmed allelopathic activity by important crops including rye (Secale cereale) and wheat (Triticum aestivum) Belz, 2004), barley (Hordeum vulgare) (Liu \& Lovett, 1993), sorghum (Sorghum bicolor) (Weston et al., 2013), and sunflower (Helianthus annuus) (Jabran et al., 2015). Thus, it is evident that the potential for allelopathic activity is present in rotational crops. However, limited literature is available on the allelopathic effects of either root leachates or plant residue leachates of weeds and crops, grown in the Western Cape, South Africa. Here, we used pot leachates to assess the bioactivity of these allelochemicals on rotational crop plants and ryegrass. Crop plants and ryegrass grown under controlled conditions treated with either root leachate from living plants, or corresponding plant residue leachate.

Since limited information on the relative allelopathic potential of rotational crops is available to producers, the complexity of these interactions complicates conclusive field research Wu et al., 2001). Similarly, analising these chemical compounds in a laboratory requires expensive facilities and methods not always immediately available in resource poor situations. However, plant screenings under controlled conditions allows for insight into these interactions. Subsequent to previous results on rotational crop seeds and seedlings Ferreira et al., 2018), we hypothesized that allelopathic root and plant residue leachates is involved in crop-crop and cropweed interference due to the release of leachates by root systems and leachates from decomposing plant material from the various crop species. Consequently, the initial objective was a preliminary assessment of allelopathic effects on plant growth and biomass production following treatment with root and aboveground plant residue leachates. Secondly, the aim was to evaluate whether pot leachates from roots and plant residues obtained from rotational crops and ryegrass show mutual allelopathic interactions. This information is essential to improve sustainable weed management practices in view of the increasing role of allelopathy in sustainable agriculture.

\section{MATERIALS AND METHODS}

Allelopathic experiments are often controversial due to the experimental design choice Christina et al., 2015). However, for both Experiments I and II we used research methods similar in 
concept to those followed by Smith et al. (2001) and Reinhardt et al. (1994), but it differed in terms of both experimental method and plant series investigated. This enabled assessment of the effects of pot leachates on the development and growth of crop plants. Viard-Crétat et al. (2009) modified this experimental design by involving target pots (receivers) watered by leachates from donor pots. This is an appropriate setup for studying allelopathy, since it assumes that the leachates contain the allelopathic compounds released by donor plants Christina et al., 2015). Using this design allowed us to validate our hypothesis on the involvement of allelopathy in crop-weed plant interactions due to the release of leachates by root systems and leachates from decomposing plant material from the various crop species.

The rotational plant series used in the greenhouse consisted of the rotational crops barley (Hordeum vulgare L. v. Clipper), canola (Brassica napus L. v. ATR Hyden), wheat (Triticum aestivum v. SST 88), lupine (Lupinus albus L. v. Tanjil), alfalfa (Medicago sativa L. v. SA standard), medic (Medicago truncatula Gaertn. v. Parabinga) and ryegrass (Lolium multiflorum Lam. v. Energa). All seeds were commercially standard products and obtained to ensure consistent germination under controlled conditions.

Experiment I: This experiment conducted in a glasshouse, determined the effects of pot leachate from each plant in the series on the inter- and intra-plant growth of all species. Ten crop seeds of each plant type were planted in separate pots filled with $6 \mathrm{~kg}$ of leached river sand, and thinned to five plants of similar size one week after emergence. Control pots were not seeded, but its leachate collected as above for use in treatments later on. Treatments in the greenhouse were replicated three times in a Randomised Block design and the experiment was repeated twice.

Experiment II: The second experiment conducted in a glasshouse studied the effects of aboveground plant residue leachates from the plant series on the growth of each plant mutually. Plant material from each species was collected in the field and air-dried, after which it was ground to a coarse powder. This substratum was mixed shallowly into pots filled with $6 \mathrm{~kg}$ of leached river sand. A substratum rate of $15 \mathrm{~g}$ per pot (equivalent to six tons plant residues per hectare), in which the donor plant itself, as well as all species in the plant series, was planted separately (five plants per pot), was utilised. Pots without plant residue incorporation were utilised as control, with its leachate collected as above for use as treatments later on. Treatments in the greenhouse were replicated three times in a Randomised Block design and the experiment repeated twice.

Twice a week until five weeks after emergence, all pots in both experiments were irrigated to above soil field capacity, from the first week after planting to provide at least $100 \mathrm{ml}$ leachate per pot. Leachates from all pots collected in designated containers, was utilised in treatments. Once a week, $100 \mathrm{ml}$ Multifeed Classic Fertiliser ${ }^{\circledR}$ was applied as a balanced plant nutrition at a concentration of $1 \mathrm{~g} \ell^{-1}$, to each pot to maintain plant health.

In the greenhouse, plant height was determined for all plants on a weekly basis, starting at one week after emergence. After five weeks, all plants were cut off at ground level. Thereafter, all the aboveground plant parts were dried at $60^{\circ} \mathrm{C}$ for 72 hours and the dry mass recorded. 
All data were analysed statistically (ANOVA) with the statistical program SAS [SAS Institute Inc., Cary, NC, USA]. Least significant differences were used to identify significant differences between means at the $5 \%$ level of probability.

\section{RESULTS}

\subsection{Barley}

\subsubsection{Root leachates}

At three weeks after planting a significant reduction in barley plant height, was recorded after treatment with leachates from the root systems of both alfalfa and medic (Table 1). This significant growth-inhibiting effect from alfalfa and medic on barley, along with lupine, was also evident at five weeks after planting. The dry mass of barley, treated with wheat, lupine and alfalfa root leachates, was significantly less than the control.

Table 1 Effects of root and aboveground leachates on barley plant height and dry mass in a greenhouse

\begin{tabular}{|l|l|l|l|l|l|l|}
\hline & \multicolumn{3}{|l|}{ Root leachates } & \multicolumn{3}{l|}{ Aboveground leachates } \\
\hline Plant type & $\begin{array}{l}\text { Barley } \\
\text { plant } \\
\text { height at } \\
\text { wks (cm) }\end{array}$ & $\begin{array}{l}\text { Barley } \\
\text { plant } \\
\text { height at } \\
\text { wks (cm) }\end{array}$ & $\begin{array}{l}\text { Barley dry } \\
\text { mass (g) }\end{array}$ & $\begin{array}{l}\text { Barley } \\
\text { lant } \\
\text { height at 3 } \\
\text { wks (cm) }\end{array}$ & $\begin{array}{l}\text { Barley } \\
\text { plant } \\
\text { height at 5 } \\
\text { wks (cm) }\end{array}$ & $\begin{array}{l}\text { Barley dry } \\
\text { mass (g) }\end{array}$ \\
\hline Barley & $38.6 \mathrm{a}$ & $46.9 \mathrm{ab}$ & $0.75 \mathrm{a}$ & $25 \mathrm{c}$ & $36.9 \mathrm{~b}$ & $2.09 \mathrm{bc}$ \\
\hline Canola & $37.5 \mathrm{ab}$ & $47.2 \mathrm{a}$ & $0.61 \mathrm{bcd}$ & $27.7 \mathrm{abc}$ & $38 \mathrm{ab}$ & $1.56 \mathrm{bc}$ \\
\hline Wheat & $37.6 \mathrm{ab}$ & $43.9 \mathrm{bc}$ & $0.58 \mathrm{~cd}$ & $29.3 \mathrm{abc}$ & $41.6 \mathrm{ab}$ & $3.97 \mathrm{a}$ \\
\hline Lupine & $35.7 \mathrm{bc}$ & $42.6 \mathrm{c}$ & $0.56 \mathrm{~d}$ & $30.3 \mathrm{abc}$ & $42.2 \mathrm{ab}$ & $2.35 \mathrm{bc}$ \\
\hline Alfalfa & $31.2 \mathrm{~d}$ & $38.6 \mathrm{~d}$ & $0.54 \mathrm{~d}$ & $33.7 \mathrm{ab}$ & $46.9 \mathrm{a}$ & $1.44 \mathrm{bc}$ \\
\hline Medic & $34.3 \mathrm{c}$ & $42.3 \mathrm{c}$ & $0.63 \mathrm{bcd}$ & $34.4 \mathrm{a}$ & $44.8 \mathrm{ab}$ & $1.36 \mathrm{c}$ \\
\hline Ryegrass & $37.3 \mathrm{ab}$ & $45.3 \mathrm{abc}$ & $0.67 \mathrm{abc}$ & $32.8 \mathrm{ab}$ & $41.1 \mathrm{ab}$ & $1.69 \mathrm{bc}$ \\
\hline Control & $38.1 \mathrm{ab}$ & $46.6 \mathrm{ab}$ & $0.69 \mathrm{ab}$ & $26.4 \mathrm{bc}$ & $36.7 \mathrm{~b}$ & $2.42 \mathrm{~b}$ \\
\hline LSD (P $\leq 0.05)$ & 2.4 & 3.2 & 0.11 & 7.7 & 9.4 & 1.04 \\
\hline
\end{tabular}

Means followed by the same letter are not significantly different at the 0.05 probability level

\subsubsection{Aboveground plant residue leachates}

Leachate from medic plant residues increased barley plant height significantly at three weeks after planting (Table 1). At five weeks after planting, leachate from alfalfa had stimulated barley height significantly from that attained at the control treatment. Treatment with leachate from wheat plant residues, caused barley dry mass to increase significantly compared to the control. 
Vol. 06, No. 05; 2021

ISSN: $2456-8643$

By contrast, the dry mass of barley treated with leachate from medic residues, reduced significantly.

\subsection{Canola}

\subsubsection{Root leachates}

Treatment with root leachates from barley, canola, alfalfa, medic and ryegrass, caused canola plant height to be significantly greater at five weeks after planting (Table 2).

\subsubsection{Aboveground plant residue leachates}

Aboveground leachates from alfalfa, medic and ryegrass significantly increased canola plant height from that attained at the control at three weeks after planting (Table 2). Similarly, at five weeks after planting, leachates from alfalfa, medic and ryegrass had stimulated canola height significantly compared to the control. Also, the dry mass of canola treated with the aboveground leachate from wheat was significantly greater than the control.

Table 2 Effects of root and aboveground leachates on canola plant height and dry mass in a greenhouse

\begin{tabular}{|l|l|l|l|l|l|l|}
\hline & \multicolumn{3}{|l|}{ Root leachates } & \multicolumn{3}{l|}{ Aboveground leachates } \\
\hline & $\begin{array}{l}\text { Canola } \\
\text { plant } \\
\text { height at } \\
\text { wks (cm) }\end{array}$ & $\begin{array}{l}\text { Canola } \\
\text { plant } \\
\text { height at } \\
\text { wks (cm) }\end{array}$ & $\begin{array}{l}\text { Canola dry } \\
\text { mass (g) }\end{array}$ & $\begin{array}{l}\text { Canola } \\
\text { plant } \\
\text { height at 3 } \\
\text { wks (cm) }\end{array}$ & $\begin{array}{l}\text { Canola } \\
\text { plant } \\
\text { height at 5 } \\
\text { wks (cm) }\end{array}$ & $\begin{array}{l}\text { Canola dry } \\
\text { mass (g) }\end{array}$ \\
\hline Barley & $12.5 \mathrm{a}$ & $21.9 \mathrm{a}$ & $0.67 \mathrm{ab}$ & $5.5 \mathrm{~d}$ & $12.6 \mathrm{c}$ & $2.52 \mathrm{~b}$ \\
\hline Canola & $13.7 \mathrm{a}$ & $20.5 \mathrm{ab}$ & $0.70 \mathrm{ab}$ & $7.0 \mathrm{bcd}$ & $14.2 \mathrm{bc}$ & $2.23 \mathrm{bc}$ \\
\hline Wheat & $12.3 \mathrm{a}$ & $18.8 \mathrm{bc}$ & $0.60 \mathrm{~b}$ & $7.1 \mathrm{bcd}$ & $14.8 \mathrm{abc}$ & $4.34 \mathrm{a}$ \\
\hline Lupine & $12.9 \mathrm{a}$ & $19.1 \mathrm{bc}$ & $0.63 \mathrm{~b}$ & $5.7 \mathrm{~cd}$ & $15.2 \mathrm{abc}$ & $2.19 \mathrm{bc}$ \\
\hline Alfalfa & $12.4 \mathrm{a}$ & $20.3 \mathrm{ab}$ & $0.65 \mathrm{~b}$ & $9.3 \mathrm{a}$ & $17.2 \mathrm{ab}$ & $1.35 \mathrm{c}$ \\
\hline Medic & $13.1 \mathrm{a}$ & $21.7 \mathrm{a}$ & $0.71 \mathrm{ab}$ & $8.1 \mathrm{ab}$ & $17.4 \mathrm{a}$ & $1.41 \mathrm{bc}$ \\
\hline Ryegrass & $13.1 \mathrm{a}$ & $21.7 \mathrm{a}$ & $0.77 \mathrm{a}$ & $7.7 \mathrm{abc}$ & $15.7 \mathrm{ab}$ & $1.58 \mathrm{bc}$ \\
\hline Control & $12.7 \mathrm{a}$ & $18.1 \mathrm{c}$ & $0.67 \mathrm{ab}$ & $5.5 \mathrm{~d}$ & $12.3 \mathrm{c}$ & $1.77 \mathrm{bc}$ \\
\hline LSD (P $\leq 0.05)$ & 1.7 & 2.1 & 0.12 & 2.1 & 3.1 & 2.82 \\
\hline
\end{tabular}

Means followed by the same letter are not significantly different at the 0.05 probability level

\subsection{Wheat}

\subsubsection{Root leachates}

A significant growth stimulating effect by ryegrass root leachate on wheat plant height was evident at both three and five weeks after planting (Table 3). 
Table 3 Effects of root and aboveground leachates on wheat plant height and dry mass in a greenhouse

\begin{tabular}{|l|l|l|l|l|l|l|}
\hline & \multicolumn{3}{|l|}{ Root leachates } & \multicolumn{3}{l|}{ Aboveground leachates } \\
\hline Plant type & $\begin{array}{l}\text { Wheat } \\
\text { plant } \\
\text { height at } \\
\text { wks (cm) }\end{array}$ & $\begin{array}{l}\text { Wheat } \\
\text { plant } \\
\text { height at } 5 \\
\text { wks (cm) }\end{array}$ & $\begin{array}{l}\text { Wheat dry } \\
\text { mass (g) }\end{array}$ & $\begin{array}{l}\text { Wheat } \\
\text { plant } \\
\text { height at 3 } \\
\text { wks (cm) }\end{array}$ & $\begin{array}{l}\text { Wheat } \\
\text { plant } \\
\text { height at 5 } \\
\text { wks (cm) }\end{array}$ & $\begin{array}{l}\text { Wheat dry } \\
\text { mass (g) }\end{array}$ \\
\hline Barley & $34.6 \mathrm{ab}$ & $45.0 \mathrm{ab}$ & $0.96 \mathrm{ab}$ & $29.1 \mathrm{bc}$ & $33.4 \mathrm{c}$ & $2.67 \mathrm{~cd}$ \\
\hline Canola & $33.2 \mathrm{bc}$ & $45.7 \mathrm{ac}$ & $0.87 \mathrm{ab}$ & $26.9 \mathrm{bc}$ & $37.6 \mathrm{c}$ & $1.89 \mathrm{de}$ \\
\hline Wheat & $34.1 \mathrm{bc}$ & $44.3 \mathrm{ab}$ & $0.93 \mathrm{ab}$ & $28.9 \mathrm{bc}$ & $37.6 \mathrm{c}$ & $4.67 \mathrm{a}$ \\
\hline Lupine & $32.1 \mathrm{c}$ & $42.8 \mathrm{~b}$ & $0.77 \mathrm{~b}$ & $28.8 \mathrm{bc}$ & $36.3 \mathrm{c}$ & $3.46 \mathrm{bc}$ \\
\hline Alfalfa & $32.5 \mathrm{bc}$ & $43.5 \mathrm{ab}$ & $0.87 \mathrm{ab}$ & $30.3 \mathrm{~b}$ & $36.5 \mathrm{c}$ & $0.93 \mathrm{e}$ \\
\hline Medic & $34.7 \mathrm{ab}$ & $44.9 \mathrm{ab}$ & $1.00 \mathrm{a}$ & $37.0 \mathrm{a}$ & $46.5 \mathrm{a}$ & $1.01 \mathrm{e}$ \\
\hline Ryegrass & $36.4 \mathrm{a}$ & $45.8 \mathrm{a}$ & $0.97 \mathrm{a}$ & $34.0 \mathrm{a}$ & $38.7 \mathrm{bc}$ & $1.8 \mathrm{de}$ \\
\hline Control & $33.5 \mathrm{bc}$ & $43.1 \mathrm{ab}$ & $0.89 \mathrm{ab}$ & $26.5 \mathrm{c}$ & $44.6 \mathrm{ab}$ & $3.94 \mathrm{ab}$ \\
\hline LSD (P $\leq 0.05)$ & 2.3 & 2.9 & 0.19 & 3.4 & 6.4 & 1.06 \\
\hline
\end{tabular}

Means followed by the same letter are not significantly different at the 0.05 probability level

\subsubsection{Aboveground plant residue leachate}

Alfalfa, medic and ryegrass aboveground leachates increased wheat plant height significantly more than that attained at the control at three weeks after planting (Table 3). In contrast, at five weeks after planting, leachates from barley, canola, wheat, lupine and alfalfa had inhibited wheat height significantly. The dry mass of wheat treated with barley, canola, alfalfa, medic and ryegrass aboveground leachates, was significantly less than the control.

\subsection{Lupine}

\subsubsection{Root leachates}

Stimulation of lupine plant height from root leachates of barley was significant at three weeks after planting. This growth-stimulating effect from barley, in addition to that of medic and ryegrass root leachates, was also evident in lupine plant height at five weeks after planting (Table 4).

\subsubsection{Aboveground plant residue leachates}


No significant differences between treatments were recorded in lupine plant height at either three or five weeks, or in dry mass (Table 4).

Table 4 Effects of root exudates and above-ground plant residue leachates on lupine plant height and dry mass

\begin{tabular}{|c|c|c|c|c|c|c|}
\hline & \multicolumn{3}{|c|}{ Root leachate } & \multicolumn{3}{|c|}{ Above-ground leachate } \\
\hline Plant type & $\begin{array}{l}\text { Lupine } \\
\text { plant } \\
\text { height at } 3 \\
\text { wks }(\mathrm{cm})\end{array}$ & $\begin{array}{l}\text { Lupine } \\
\text { plant } \\
\text { height at } 5 \\
\text { wks (cm) }\end{array}$ & $\begin{array}{l}\text { Lupine dry } \\
\text { mass (g) }\end{array}$ & $\begin{array}{l}\text { Lupine } \\
\text { plant } \\
\text { height at } 3 \\
\text { wks (cm) }\end{array}$ & $\begin{array}{l}\text { Lupine } \\
\text { plant } \\
\text { height at } 5 \\
\text { wks (cm) }\end{array}$ & $\begin{array}{l}\text { Lupine dry } \\
\text { mass }(\mathrm{g})\end{array}$ \\
\hline Barley & $18.6 \mathrm{a}$ & $29.9 \mathrm{ab}$ & $0.8 \mathrm{ab}$ & $17.8 \mathrm{ab}$ & $24.1 \mathrm{ab}$ & $2.61 \mathrm{ab}$ \\
\hline Canola & $17.3 \mathrm{ab}$ & $28.3 \mathrm{abc}$ & $0.82 \mathrm{ab}$ & $9.0 \mathrm{~b}$ & $16.2 \mathrm{~b}$ & $2.31 \mathrm{abc}$ \\
\hline Wheat & $17.7 \mathrm{ab}$ & $28.1 \mathrm{bc}$ & $0.86 \mathrm{a}$ & $19.1 \mathrm{a}$ & $31.8 \mathrm{a}$ & $3.48 \mathrm{a}$ \\
\hline Lupine & $16.2 \mathrm{bc}$ & $27.1 \mathrm{bc}$ & $0.87 \mathrm{a}$ & $9.8 \mathrm{~b}$ & $16.0 \mathrm{~b}$ & $1.77 \mathrm{bc}$ \\
\hline Lucerne & $14.1 \mathrm{c}$ & $25.7 \mathrm{c}$ & $0.73 \mathrm{~b}$ & $20.6 \mathrm{a}$ & $29.4 a b$ & $0.87 \mathrm{c}$ \\
\hline Medic & $16.3 \mathrm{abc}$ & $31.3 \mathrm{a}$ & $0.87 \mathrm{a}$ & $14.7 \mathrm{ab}$ & $27.4 \mathrm{ab}$ & $1.07 \mathrm{bc}$ \\
\hline Ryegrass & $17.1 \mathrm{ab}$ & $29.7 \mathrm{ab}$ & $0.84 \mathrm{a}$ & $14.9 \mathrm{ab}$ & $25.8 \mathrm{ab}$ & $2.06 a b c$ \\
\hline Control & $15.5 \mathrm{bc}$ & $25.9 \mathrm{c}$ & $0.82 \mathrm{ab}$ & $12.7 \mathrm{ab}$ & $22.1 \mathrm{ab}$ & $1.90 \mathrm{abc}$ \\
\hline $\operatorname{LSD}(\mathrm{P} \leq 0.05)$ & 5.5 & 3.2 & 0.1 & 9 & 14.3 & 1.64 \\
\hline
\end{tabular}

Means followed by the same letter are not significantly different at the 0.05 probability level

\subsection{Alfalfa}

\subsubsection{Root leachates}

Compared to the control, barley root leachate showed significant stimulatory activity on alfalfa shoot length, at three weeks after planting (Table 4).

\subsubsection{Aboveground plant residue leachates}

At three weeks after planting, aboveground leachates from barley, alfalfa, medic and ryegrass had stimulated alfalfa shoot length significantly from that attained at the control treatment (Table 5). However, at five weeks after planting, only medic leachate increased alfalfa shoot length significantly from the control. This growth-stimulating effect was also evident in alfalfa dry mass after treatment with barley, canola, wheat, lupine, medic and ryegrass leachates.

Table 5 Effects of root and aboveground leachates on alfalfa shoot length and dry mass in a greenhouse 


\section{International Journal of Agriculture, Environment and Bioresearch}

Vol. 06, No. 05; 2021

ISSN: $2456-8643$

\begin{tabular}{|l|l|l|l|l|l|l|}
\hline & \multicolumn{4}{|l|}{ Root leachates } & \multicolumn{3}{l|}{ Aboveground leachates } \\
\hline Plant type & $\begin{array}{l}\text { Alfalfa } \\
\text { shoot } \\
\text { length at } \\
\text { wks (cm) }\end{array}$ & $\begin{array}{l}\text { Alfalfa } \\
\text { shoot } \\
\text { length at } \\
\text { wks (cm) }\end{array}$ & $\begin{array}{l}\text { Alfalfa } \\
\text { dry mass } \\
\text { g) }\end{array}$ & $\begin{array}{l}\text { Alfalfa } \\
\text { shoot } \\
\text { length at } \\
\text { wks (cm) }\end{array}$ & $\begin{array}{l}\text { Alfalfa } \\
\text { shoot } \\
\text { length at 5 5 } \\
\text { wks (cm) }\end{array}$ & $\begin{array}{l}\text { Alfalfa } \\
\text { dry mass } \\
\text { g) }\end{array}$ \\
\hline Barley & $11.7 \mathrm{a}$ & $26.3 \mathrm{a}$ & $0.31 \mathrm{a}$ & $9.0 \mathrm{a}$ & $25.7 \mathrm{ab}$ & $3.33 \mathrm{bc}$ \\
\hline Canola & $9.5 \mathrm{~b}$ & $20.7 \mathrm{abc}$ & $0.24 \mathrm{a}$ & $8.2 \mathrm{ab}$ & $23.3 \mathrm{ab}$ & $2.62 \mathrm{~cd}$ \\
\hline Wheat & $8.6 \mathrm{~b}$ & $18.1 \mathrm{bc}$ & $0.20 \mathrm{a}$ & $8.4 \mathrm{ab}$ & $22.4 \mathrm{ab}$ & $4.81 \mathrm{a}$ \\
\hline Lupine & $9.8 \mathrm{ab}$ & $19.5 \mathrm{abc}$ & $0.30 \mathrm{a}$ & $4.7 \mathrm{c}$ & $16.9 \mathrm{~b}$ & $4.21 \mathrm{ab}$ \\
\hline Alfalfa & $7.9 \mathrm{~b}$ & $17.5 \mathrm{c}$ & $0.32 \mathrm{a}$ & $10.3 \mathrm{a}$ & $22.8 \mathrm{ab}$ & $1.67 \mathrm{de}$ \\
\hline Medic & $9.5 \mathrm{~b}$ & $24.9 \mathrm{ab}$ & $0.32 \mathrm{a}$ & $9.2 \mathrm{a}$ & $28.7 \mathrm{a}$ & $2.12 \mathrm{~d}$ \\
\hline Ryegrass & $9.8 \mathrm{ab}$ & $23.7 \mathrm{abc}$ & $0.25 \mathrm{a}$ & $10 \mathrm{a}$ & $22.5 \mathrm{ab}$ & $3.11 \mathrm{c}$ \\
\hline Control & $8.5 \mathrm{~b}$ & $21.1 \mathrm{abc}$ & $0.30 \mathrm{a}$ & $5.5 \mathrm{bc}$ & $17.3 \mathrm{~b}$ & $0.74 \mathrm{e}$ \\
\hline $\begin{array}{l}\text { LSD } \\
\text { (P } \leq 0.05)\end{array}$ & 2.1 & 7.1 & 0.18 & 3.4 & 10 & 0.96 \\
\hline
\end{tabular}

Means followed by the same letter are not significantly different at the 0.05 probability level

\subsection{Medic}

Table 6 Effects of root and aboveground leachates on medic shoot length and dry mass in a greenhouse

\begin{tabular}{|l|l|l|l|l|l|l|}
\hline & \multicolumn{3}{|l|}{ Root leachates } & \multicolumn{3}{l|}{ Aboveground leachates } \\
\hline Plant type & $\begin{array}{l}\text { Medic } \\
\text { shoot } \\
\text { length at 3 } \\
\text { wks (cm) }\end{array}$ & $\begin{array}{l}\text { Medic } \\
\text { shoot } \\
\text { length at 5 } \\
\text { wks (cm) }\end{array}$ & $\begin{array}{l}\text { Medic dry } \\
\text { mass (g) }\end{array}$ & $\begin{array}{l}\text { Medic } \\
\text { shoot } \\
\text { length at 3 3 } \\
\text { wks (cm) }\end{array}$ & $\begin{array}{l}\text { Medic } \\
\text { shoot } \\
\text { length at 5 } \\
\text { wks (cm) }\end{array}$ & $\begin{array}{l}\text { Medic dry } \\
\text { mass (g) }\end{array}$ \\
\hline Barley & $7.5 \mathrm{ab}$ & $15.5 \mathrm{ab}$ & $0.40 \mathrm{~b}$ & $5.8 \mathrm{ab}$ & $11.9 \mathrm{ab}$ & $3.52 \mathrm{~b}$ \\
\hline Canola & $7.3 \mathrm{ab}$ & $12.5 \mathrm{bc}$ & $0.42 \mathrm{ab}$ & $5.8 \mathrm{ab}$ & $10.4 \mathrm{~b}$ & $3.09 \mathrm{bc}$ \\
\hline Wheat & $6.1 \mathrm{bc}$ & $10.6 \mathrm{~cd}$ & $0.41 \mathrm{~b}$ & $5.4 \mathrm{ab}$ & $11.5 \mathrm{ab}$ & $6.10 \mathrm{a}$ \\
\hline Lupine & $3.7 \mathrm{~d}$ & $7.9 \mathrm{~d}$ & $0.20 \mathrm{c}$ & $5.1 \mathrm{ab}$ & $10.4 \mathrm{~b}$ & $3.08 \mathrm{bc}$ \\
\hline
\end{tabular}


Vol. 06, No. 05; 2021

ISSN: $2456-8643$

\begin{tabular}{|l|l|l|l|l|l|l|}
\hline Alfalfa & $6.5 \mathrm{abc}$ & $15.2 \mathrm{ab}$ & $0.59 \mathrm{a}$ & $7.1 \mathrm{a}$ & $15.1 \mathrm{a}$ & $1.69 \mathrm{~cd}$ \\
\hline Medic & $5.5 \mathrm{c}$ & $14.6 \mathrm{abc}$ & $0.35 \mathrm{bc}$ & $7.2 \mathrm{a}$ & $13.5 \mathrm{ab}$ & $2.23 \mathrm{bcd}$ \\
\hline Ryegrass & $8.0 \mathrm{a}$ & $17.5 \mathrm{a}$ & $0.46 \mathrm{ab}$ & $6.1 \mathrm{ab}$ & $11.7 \mathrm{ab}$ & $2.66 \mathrm{bc}$ \\
\hline Control & $6.4 \mathrm{abc}$ & $15.2 \mathrm{ab}$ & $0.41 \mathrm{~b}$ & $3.7 \mathrm{~b}$ & $10.7 \mathrm{~b}$ & $0.73 \mathrm{~d}$ \\
\hline $\begin{array}{l}\text { LSD } \\
(\mathrm{P} \leq 0.05)\end{array}$ & 1.7 & 4 & 0.17 & 2.6 & 4.1 & 1.59 \\
\hline
\end{tabular}

Means followed by the same letter are not significantly different at the 0.05 probability level

\subsubsection{Root leachates}

Treatment with lupine root leachate significantly reduced shoot length of medic at both three and five weeks after planting (Table 6). Also at five weeks after planting, wheat root leachate inhibited medic shoot length significantly from that attained at the control. The dry mass of medic treated with lupine root leachate was significantly lower than the control, but in contrast to this, alfalfa root leachate caused a significant increase in the studied parameter.

\subsubsection{Aboveground plant residue leachates}

At three weeks after planting, leachates from alfalfa and medic had stimulated medic shoot length significantly from that attained at the control (Table 6). This growth stimulating effect from alfalfa leachates on shoot length was also evident at five weeks after planting. The dry mass of medic, treated with barley, canola, wheat, lupine and ryegrass leachates, was significantly greater than the control.

\subsection{Ryegrass}

\subsubsection{Root leachates}

Alfalfa root leachate significantly inhibited ryegrass plant height at three weeks after planting (Table 7). The dry mass of ryegrass treated with wheat and lupine root leachates was significantly higher than the control. In contrast to this, ryegrass root leachate, showed autotoxicity by significantly reducing ryegrass dry mass.

Table 7 Effects of root and aboveground leachates on ryegrass plant height and dry mass in a greenhouse

\begin{tabular}{|l|l|l|l|l|l|l|}
\hline & \multicolumn{3}{|l|}{ Root leachates } & \multicolumn{2}{l|}{ Aboveground leachates } \\
\hline & $\begin{array}{l}\text { Ryegrass } \\
\text { plant } \\
\text { height at 3 } \\
\text { wks (cm) }\end{array}$ & $\begin{array}{l}\text { Ryegrass } \\
\text { plant } \\
\text { height at 5 5 } \\
\text { wks (cm) }\end{array}$ & $\begin{array}{l}\text { Ryegrass } \\
\text { dry mass } \\
\text { (g) }\end{array}$ & $\begin{array}{l}\text { Ryegrass } \\
\text { plant } \\
\text { height at 3 } \\
\text { wks (cm) }\end{array}$ & $\begin{array}{l}\text { Ryegrass } \\
\text { plant } \\
\text { height at 5 } \\
\text { wks (cm) }\end{array}$ & $\begin{array}{l}\text { Ryegrass } \\
\text { dry mass } \\
(\mathrm{g})\end{array}$ \\
\hline Barley & $30.8 \mathrm{a}$ & $39.2 \mathrm{a}$ & $0.72 \mathrm{~b}$ & $22.5 \mathrm{ab}$ & $33.3 \mathrm{ab}$ & $2.7 \mathrm{~b}$ \\
\hline
\end{tabular}


Vol. 06, No. 05; 2021

ISSN: $2456-8643$

\begin{tabular}{|l|l|l|l|l|l|l|} 
Canola & $30.3 \mathrm{a}$ & $38.8 \mathrm{a}$ & $0.78 \mathrm{~b}$ & $21.1 \mathrm{~b}$ & $30.1 \mathrm{~b}$ & $2.29 \mathrm{bc}$ \\
\hline Wheat & $29.7 \mathrm{a}$ & $37.8 \mathrm{a}$ & $0.97 \mathrm{a}$ & $24.9 \mathrm{ab}$ & $34.3 \mathrm{ab}$ & $4.27 \mathrm{a}$ \\
\hline Lupine & $29.6 \mathrm{ab}$ & $38.2 \mathrm{a}$ & $0.97 \mathrm{a}$ & $21.7 \mathrm{ab}$ & $35.2 \mathrm{ab}$ & $2.43 \mathrm{~b}$ \\
\hline Alfalfa & $26.1 \mathrm{~b}$ & $36.5 \mathrm{a}$ & $0.86 \mathrm{ab}$ & $28.3 \mathrm{a}$ & $38.3 \mathrm{a}$ & $1.57 \mathrm{~cd}$ \\
\hline Medic & $27.4 \mathrm{ab}$ & $38.7 \mathrm{a}$ & $0.71 \mathrm{~b}$ & $24.7 \mathrm{ab}$ & $33.8 \mathrm{ab}$ & $1.47 \mathrm{~d}$ \\
\hline Ryegrass & $29.8 \mathrm{a}$ & $36.4 \mathrm{a}$ & $0.56 \mathrm{c}$ & $23.1 \mathrm{ab}$ & $33.0 \mathrm{ab}$ & $1.99 \mathrm{bcd}$ \\
\hline Control & $30.9 \mathrm{a}$ & $40.5 \mathrm{a}$ & $0.78 \mathrm{~b}$ & $20.7 \mathrm{~b}$ & $35.5 \mathrm{ab}$ & $1.98 \mathrm{bcd}$ \\
\hline LSD (P $\leq 0.05)$ & 3.5 & 4.4 & 0.15 & 2.8 & 5.6 & 0.78 \\
\hline
\end{tabular}

Means followed by the same letter are not significantly different at the 0.05 probability level

\subsubsection{Aboveground plant residue leachates}

Stimulation of ryegrass plant height was significant from that attained at the control at three weeks after planting, following treatment with aboveground leachate from alfalfa (Table 7). Similarly, the increase in ryegrass dry mass was significant after treatment with wheat above ground leachate.

\section{DISCUSSION}

Results obtained proved the hypothesis that these crop plants and ryegrass contain allelopathic compounds exhibiting both phyto-inhibitory and stimulatory effects on the test plants. The observed differential plant growth inhibition and stimulation for both root leachates and aboveground leachates, suggest the presence of different allelochemicals in various amounts in test plants. However, the degree of inhibition and stimulation varied greatly amongst the different crops and ryegrass with aboveground plant residue leachates showing the greatest phytotoxic activity. Overall, among all studied parameters, the leguminous crops, namely lupine, alfalfa and medic caused the most significant responses. Henceforth, the following discussion includes only significant interactions of the studied parameters observed twice for a test plant.

Plant dry mass production of barley was reduced by both lupine (-19\%) and alfalfa (-22\%). At both three and five weeks, root leachates from alfalfa $(-18 \%$ and $-17 \%)$ and medic $(-10 \%$ and 9\%) caused a reduction in barley plant height. This result is in accordance with studies on alfalfa by Gholami et al. (2014) that indicated allelopathic triterpene, saponins and flavonoids as the major cause of growth inhibition and yield reduction in subsequent crops. In contrast, leachates from aboveground plant residues from alfalfa at five weeks $(+28 \%)$ and medic at three weeks $(+30 \%)$ stimulated barley growth in terms of plant height. This result may be explained by the hormesis effect, since the same allelochemicals caused different growth responses, either positive or negative, from the species considered here. Furthermore, the span between stimulation and inhibition for allelochemicals can be small and hormetic effects (Calabrese, 2007) may occur in a natural setting if doses released are low (Belz, 2008). 
Vol. 06, No. 05; 2021

ISSN: $2456-8643$

Similar to barley, wheat growth at three weeks was stimulated by leachates from aboveground plant residues from alfalfa $(+14 \%)$ and medic $(+40 \%)$ as well as ryegrass $(+28 \%)$. Also, root leachates from ryegrass stimulated wheat growth at three weeks $(+9 \%)$. Contrastingly, aboveground barley $(-25 \%)$, canola $(-16 \%)$ and alfalfa $(-18 \%)$ leachates reduced wheat growth at five weeks. In addition, wheat dry mass production was reduced by aboveground leachates from barley $(-32 \%)$, canola $(-52 \%)$, alfalfa $(-76 \%)$ and ryegrass $(-54 \%)$. Thus, a transition from stimulatory to inhibitory effects over time was observed for aboveground ryegrass residue leachates. According to Kruidhof (2008) there are two possible explanations for this. Firstly, it is widely recognized that low concentrations of allelochemicals can be stimulating to weed germination and early growth (Belz, 2004). Secondly, the observed stimulation could be a response to increased nutrient and especially nitrate levels in the residue-amended soil, because nitrate stimulates weed seed germination (Kruidhof, 2008). These results are in close conformity with results by Siyar et al. (2019) on wheat parameters in the presence of aqueous extracts of weeds. Also Sturm et al. (2018) reported that another monocotyledonous species, Avena strigosa, reduced the growth of wheat by between $48 \%$ and $67 \%$ and concluded that it showed significant allelopathic effects on wheat. It is inferred that reduced plant height of wheat at the applied volumes of leachates of different plants in this experiment, might be due to the presence of phytotoxic compounds in those plants. This is also confirmed by findings of Amini et al. (2009) that the root and shoot length of wheat was reduced when grown in close proximity to ryegrass. Inhibition was greater on wheat roots than on wheat shoots. Bouhaouel et al. (2014) noted that barley allelochemicals were more toxic over time. This corresponds with results from the present study, since aboveground barley leachates did not cause a significant response in wheat growth at three weeks, but increased plant height at five weeks and also increased dry mass production. Furthermore, Bouhaouel et al. (2014) speculated that over time, barley releases different allelochemicals.

Alfalfa $(+12 \%)$, medic $(+20 \%)$ and ryegrass $(+20 \%)$ root leachates stimulated canola plant height at five weeks. For aboveground plant leachates this was even more pronounced with alfalfa $(+7 \%$ and $40 \%)$, medic $(+47 \%$ and $42 \%)$ and ryegrass $(+40 \%$ and $+28 \%)$ increasing canola plant height at both three and five weeks. An increase in lupine plant height at three $(+20 \%)$ and five $(+15 \%)$ weeks was recorded following treatment with barley root leachates. These results corroborate a pot experiment by Wynne et al. (2019) who reported no negative impacts on canola from wheat residues.

Barley root leachates stimulated alfalfa growth at three (+38\%) weeks. Similarly, aboveground barley leachates increased alfalfa shoot length at five (+64\%) weeks. This contradicts findings by Bouhaouel et al. (2014) who reported that barley allelochemicals reduced both radicle and coleoptile lengths of two monocotyledonous weeds, namely Bromus diandrus and Lolium rigidum. Bouhaouel et al. (2016) concluded that barley allelochemicals have an impact on root architecture, i.e. changes in total root length and degrees of branching when compared to the untreated seedlings. 
Vol. 06, No. 05; 2021

ISSN: $2456-8643$

Medic shoot length was inhibited by lupine root leachates at three weeks (-42\%), five weeks ($48 \%)$ and in terms of plant dry mass production $(-21 \%)$. This response is indicative of not only leguminous autotoxicity, but also confirmation that the leachates and leachates of leguminous plants utilised, possess the most phytotoxic allelochemicals. These results are in close conformity with a study by Valenzuela et al. (2018) who documented reduced root elongation of Elymus repens and stunted Arabidopsis thaliana root growth following treatment with lupine extracts. In contrast, aboveground leachates from alfalfa had stimulated medic shoot length at three $(+92 \%)$ and five weeks $(+41 \%)$ after planting.

Alfalfa root leachates caused a reduction in ryegrass plant height $(-16 \%)$ at three weeks. This confirms results by Zubair et al. (2017) who reported that some alfalfa genotypes inhibited ryegrass seed germination and reduced the root length of ryegrass seedlings by between $3 \%$ and $65 \%$. Also, the growth of ryegrass decreased in response to increasing density of alfalfa seedlings. Reduction in seed germination of annual ryegrass occurred in the presence of several alfalfa genotypes (Zubair et al., 2017). The inhibitory effect of leguminous crops on the growth and development of weeds relate to their allelopathic properties (Kocira et al., 2020). Furthermore, the inhibitory effect of alfalfa on weeds was proportional to the number and quantity of growth inhibitors (phenolic compounds) which showed strong allelopathic activity (Kocira et al., 2020). In sharp contrast, aboveground leachates from alfalfa stimulated ryegrass plant height $(+37 \%)$ at three weeks. In similar fashion, wheat root leachates $(+24 \%)$ and aboveground leachates $(+116 \%)$ increased ryegrass dry mass production. This is in disagreement with results by Shekoofa et al. (2020) that wheat suppresses germination and radicle elongation of several species.

Although only one canola variety was tested, the response of ryegrass in the current study contradicts a conclusion by Asaduzzaman et al. (2014) that highly allelopathic canola genotypes have the potential for ryegrass suppression in integrated weed management programs. Results from the current study are also in disagreement with those of Bouhaouel et al., (2014) who reported that the root allelochemicals from barley had a significant inhibitory activity on ryegrass root growth. Considering that a commercial pasture type of ryegrass (L. multiflorum Lam. v. Energa) was utilised in this study in order to ensure one seed source and consistent germination, results from a field experiment (Ferreira \& Reinhardt, 2010) showed no significant responses between this species and the hybrid weed type (Lolium multiflorum $\mathrm{x}$ L. perenne).

In the current study, the different root leachates and aboveground leachates showed variable inhibitory or stimulatory activity suggesting differences in concentrations of potent allelochemicals in the different plant parts or different active compounds. This confirms findings by Bouhaouel et al. (2014) that allelochemicals change over time. Furthermore, results showed that different plant parts exhibited differential phytotoxic activity on the test plants with the aboveground leachates having higher phytotoxicity than root leachates. This is in agreement with Chon et al. (2005) who reported that inhibition or stimulation of seedlings following treatment with plant leachates is due to the presence of allelochemicals. Results also showed that root leachates from canola exhibited no response on wheat growth whereas its aboveground leachate was more phytotoxic to wheat plant height. Consistent with current results, Ali (2016) reported 
Vol. 06, No. 05; 2021

ISSN: $2456-8643$

inhibition of annual grasses including wheat and barley using aqueous root extracts of radish (Raphanus sativus).

The differential allelopathic behaviour of the plants under evaluation in terms of inhibition or stimulation of the growth parameters reflects the presence of different allelochemicals in various amounts. The root leachates and aboveground residue leachate of plants utilised, differentially suppressed or stimulated plant growth or dry mass production. The inhibition or stimulation of plant growth observed might be due to the presence of allelochemicals that were released by the root leachates and aboveground residue leachates. These findings confirm the allelopathic suppression or stimulation of plants observed in earlier experiments on seeds and seedlings (Ferreira et al., 2018).

Aboveground leachates exhibiting higher growth inhibition suggest the presence of more potent allelochemicals in leaves and shoots than in roots. Gulzar and Sidiquie (2010) reported that foliar extracts were generally more potent than stem and root extracts, probably due to greater metabolic activity in the foliage. The fact that medic aboveground leachate inhibited barley or wheat dry mass production, but not that of canola suggest the presence of allelochemicals with a narrower spectrum of activity than the root leachates of this plant. The inhibitory activity of medic may be attributed to the presence of phenolic acids that were earlier isolated from tissues of this leguminous crop (Santos et al., 2010). Furthermore, current results showed that barley and wheat exhibited the greatest sensitivity to inhibition. This is consistent with a report by Sturm et al. (2018) on species-specific allelopathic effects.

Current results imply that allelopathic effects are complex in the field and highly context dependent. Soil characteristics such as soil nutrient status and $\mathrm{pH}$ also affect allelopathic effects of plants by influencing allelochemicals, seed germination and growth of target species ( $\mathrm{Li}$ et al., 2015). Understanding the mechanisms of and the effects that environmental stimuli have on the magnitude and type of root exudation will ultimately improve our knowledge of processes determining soil $\mathrm{CO}_{2}$ emissions, ecosystem functioning, and how to improve the sustainability of agricultural production (Canarini et al., 2019). Gfeller et al. (2013) reported on the possible effect of volatile organic compounds, known to participate in plant communication and interactions. In addition, Bouhaouel et al. (2014) speculated that the time-course evolution of the exudates and leachates after its release from the donor, leads to the decay of the allelochemicals over time and the possible higher or lower allelopathic activities. Lastly, we acknowledge that microorganisms present in the different leachates from donor pots might have modified the allelochemicals utilised for treatments. According to Christina et al. (2015) it is still unclear whether the mechanisms involving microorganisms are targets in the allelopathic process or only interfering organisms from the soil.

Nevertheless, the prominent allelopathic activity demonstrated by lupine, alfalfa and medic leachates in the current research under controlled conditions, corresponds to results obtained in the field (Ferreira \& Reinhardt, 2010) and confirm that consideration be given to these leguminous crops as cultivated and terminated mulches for weed suppression. However, such a practice is likely to be exposed to the vagaries of environmental factors, as well as likely being crop and weed-specific. Foley et al. (2011) suggested that understanding the role of root exudates in plant recognition and interference is likely to have significant impacts on agricultural 
productivity. Modern agriculture calls for sustainable systems that can maintain productivity while reducing inputs and production losses and increasing the biodiversity of agro-ecosystems (Foley et al., 2011). Furthermore, Canarini et al. (2019) concluded that root exudation is an important process determining plant interactions with the soil environment and concurred that many studies have linked this process to soil nutrient mobilization.

\section{CONCLUSIONS}

Allelopathy plays an important role in investigations on sustainable agricultural systems as well as in the suppression of weeds. The current preliminary assessment indicated that lupine, alfalfa and medic contain possible allelochemicals that might be responsible for the inhibition or stimulation exhibited on the growth and biomass production of the selected plant series. Leachate of these leguminous crops were more efficient in growth inhibition or stimulation and dry mass production than leachates from barley, canola, wheat and ryegrass. Furthermore, mulches of these crop plants that suppress weeds could reduce dependency on herbicides, in particular those products that are associated with the development of weed resistance. In the case of the mulch being a leguminous plant, biological nitrogen fixation is an added benefit. Future research should elucidate the specific allelopathic compounds in these crop plants and its use as mulches that suppress weeds.

Author Contributions: Conceptualization, M.I. Ferreira and C.F. Reinhardt; methodology, C.F. Reinhardt; software, M. van der Rijst; validation, M.I. Ferreira, C.F. Reinhardt and M. van der Rijst; formal analysis, M. van der Rijst; investigation, M.I. Ferreira; resources, M.I. Ferreira; data curation, M.I. Ferreira; writing - original draft preparation, M.I. Ferreira; writing-review and editing, C.F. Reinhardt; visualization, M.I. Ferreira and C.F. Reinhardt; supervision, C.F. Reinhardt; project administration, M.I. Ferreira; funding acquisition, M.I. Ferreira. All authors have read and agreed to the published version of the manuscript.

Funding: Grain South Africa and the Western Cape Department of Agriculture: Directorate Plant Sciences funded this research.

Conflicts of Interest: The authors declare no conflict of interest. The funders had no role in the design of the study; in the collection, analyses, or interpretation of data; in the writing of the manuscript, or in the decision to publish the results.

\section{REFERENCES}

Ali, K.A. (2016). Allelopathic potential of radish on germination and growth of some crop and weed plants. Intl Jnl Biosci 9, 394-403.

Amini, R., An, M., Pratley, J. and Azimi, S. (2009). Allelopathic assessment of annual ryegrass (Lolium rigidum): Bioassays. Allel Jnl 24, 67-76.

Amini, R.A., Movahedpour, F., Ghassemi-Golezani, K., Dabbagh Mohammadi-Nasab A. and Zafarani-Moattar P. (2012). Allelopathic assessment of common amaranth by ECAM. Intl Res Jnl App Basic Sci 3, 2268-2272.

Asaduzzaman, M., An, M., Pratley, J.E., Luckett, D.J. and Lemerle, D. (2014). Canola (Brassica napus) germplasm shows effects against annual ryegrass (Lolium rigidum). Plnt Soil 380, 47-56. DOI: $10.1007 / \mathrm{s} 11104-014-2054-4$ 
Bais, H.P., Vepachedu, R., Gilroy, S., Callaway, R.M. and Vivanco J.M. (2003). Allelopathy and exotic plant invasion: from molecules and genes to species interaction. Sci 301, 1377-1380.

Batish, D.R., Tung, P., Singh H.P. and Kohli R.K. (2002). Phytotoxicity of sunflower residues against some summer season crops. Jnl Agron Crop Sci 188, 19-24.

Belz, R.G. (2004). Evaluation of allelopathic traits in Triticum L. spp. and Secale cereale L. PhD Thesis: University of Hohenheim, Stuttgart, Germany, 157 pp.

Belz, R.G. (2008). Stimulation versus inhibition-bioactivity of parthenin, a phytochemical from Parthenium hysterophorus L. Dose response 6, 80-96.

Belz, R.G., Duke, S.O. and Hurle, K. Dose-response - a challenge for allelopathy. (2005). Nonlin Bio, Tox Med 3, 173-211.

Bouhaouel, I., Gfeller, A., Fauconnier, M.L., Delroy, B, and Du Jardin, P. (2016). Evaluation of the allelopathic potential of water-soluble compounds of barley (Hordeum vulgare L. subsp. vulgare) and great brome (Bromus diandrus Roth.) using a modified bioassay. Biotech. Agron Soc Enviro 20, 482-494.

Bouhaouel, I., Gfeller, A., Fauconnier, M.L., Rezgui, S., Amara, H. and Du Jardin, P. (2014). Allelopathic and autotoxicity effects of barley (Hordeum vulgare L. subsp. vulgare) root exudates. Biocntl 60, 425-436.

Brussaard, L., de Ruiter, P.C. and Brown, G.G. (2007). Soil biodiversity for agricultural sustainability. Agric Ecosyst Enviro 121, 233-244.

Calabrese, E.J. (2007). Biological stress response terminology: integrating the concepts of adaptive and preconditioning stress within a hormetic dose-response framework. Toxicol App Pharm 222, 122-128.

Canarini, A., Keiser C., Merchant A., Richter A. and Wanek W. (2019). Root Exudation of Primary Metabolites: Mechanisms and Their Roles in Plant Responses to Environmental Stimuli. Front Plnt Sci 10, Article 157.

Christina, M., Rouifed, S., Puijalon, S., Vallier, F., Meiffren, G., Bellvert, F. and Piola, F. (2015). Allelopathic effect of a native species on a major plant invader in Europe. The Sci Nat 102. DOI: $10.1007 / \mathrm{s} 00114-015-1263-\mathrm{x}$

Chon, S.U. Jang, H.G. Kim, D.K., Kim, Y.M., Boo, H.O. and Kim, Y.J. (2005). Allelopathic potential in lettuce (Lactuca sativa L.) Plant Sci Hort 106, 309-317.

Das, C. and Bandyopadhyay, A. (2011). Searching for allelopathic potential of Shorea robusta Gaertn.f. leaf. Bio nat 31, 29- 35.

De Gouveia Baratelli, T., Gomes A.C.C., Wessjohann L.A., Kuster R.M. and Simas, N.K. (2012). Phytochemical and allelopathic studies of Terminalia catappa L. (Combretaceae). Biocheml Syst Ecol 41, 119-125.

Ferreira, M.I. and Reinhardt, C.F. (2010). Field assessment of crop residues for allelopathic effects on both crops and weeds. Agronomy Journal102, 1593-1600.

Ferreira, M.I., Reinhardt, C.F., Lamprecht, S.C., Sinclair, M., MacKenzie, L. and Van Coller, G. (2015). Morphological identification of the ryegrass hybrid Lolium multiflorum x Lolium perenne and isolation of the pathogen Fusarium pseudograminearum in the Western Cape. S Afr Jnl Plant Soil 32, 9-15. DOI: 10.1080/02571862.2014.994140 
Ferreira, M.I., Reinhardt, C.F. and Van der Rijst, M. (2018). Assessment of the allelopathic effects of seeds and seedlings of rotational crops and ryegrass. Afr Jnl Plnt Sci 12, 309-318. DOI: $10.5897 /$ AJPS2018.17S

Foley J.A., Ramankutty N., Brauman K.A., Cassidy E.S., Gerber J.S. and Johnston M. (2011). Solutions for a cultivated planet. Nature 478, 337-342. doi: 10.1038/naturel0452

Gfeller, A. Laloux, M., Barsics, F., Kati, D.E., Haubruge, E., du Jardin P., Verheggen, F.J., Lognay, G., Wathelet, J.P. and Fauconnier, M.L. (2013). Characterization of volatile organic compounds emitted by barley (Hordeum murinum L.) roots and their attractiveness to wireworms. Jnl Chem Ecol 39, 1129-1139.

Gholami, A., De Geyter, N. and Pollier, J. (2014) Natural product biosynthesis in Medicago species. Nat Prod Repos 3, 356-380.

Gulzar, A. and Siddiqui, M.B. (2010). Allelopathic effect of aqueous extracts of different parts of Eclipta alba (L.) Hassk. on some crop and weed plants. Jnl Agric Ext Rural Dev 6, 55-60.

Jabran, K., Mahajan, G., Sardana, V. and Chauhan, B.S. (2015). Allelopathy for weed control in agricultural systems. Crop Prot 72, 57-65.

Kocira, A., Staniak, M., Tomaszewska, M., Kornas, R., Cymerman, J., Panasiewicz, K. and Lipińska, H. (2020). Legume Cover Crops as One of the Elements of Strategic Weed Management and Soil Quality Improvement. A Review. Agric 10, $394 . \quad$ DOI: 10.3390/agriculture10090394

Kruidhof, H.M. (2008). Cover crop-based ecological weed management: exploration and optimization. PhD Thesis: Wageningen University, Wageningen, The Netherlands. 156 pp.

Kumar, V., Brainard, D.C. and Bellinder, R.R. (2009). Suppression of Powell amaranth (Amaranthus powellii) by buckwheat residues: role of allelopathy. Weed Sci 57, 66-73.

Kuzyakov, Y. and Blagodatskaya, E. (2015) Microbial hotspots and hot moments in soil: concept \& review. Soil Bio Biochem 83, 184-199.

Lebecque, S., Crowet, J.M., Lins, L., Deloroy, B.M., du Jardin, P., Fauconnier, M.L. and Deleu, M. (2018). Interaction between the barley allelochemical compounds gramine and hordenine and artificial lipid bilayers mimicking the plant plasma membrane. Sci Report 8, 9784. DOI: 10.1038/s41598-018-28040-6

Li, Y., Feng, Y., Chen, Y. and Tian, Y. (2015). Soil microbes alleviate allelopathy of invasive plants. Sci Bulletin 60, 1083-1091. DOI: 10.1007/s11434-015-0819-7

Liu, D.L. and Lovett, J.V. (1993). Biologically active secondary metabolites of barley. I. Developing techniques and assessing allelopathy in barley. Jnl Chem Ecol 19, 2217-2230.

Lorenzo, P., Pereira, C.S. and Rodríguez-Echeverría, S. (2013). Differential impact on soil microbes of allelopathic compounds released by the invasive Acacia dealbata Link. Soil Biol Biochem 57, 156-163. DOI: 10.1016/j.soilbio.2012.08.018

Makoi, H.J.R. and Ndakidemi, P.A. (2012). Allelopathy as protectant, defence and growth stimulants in legume cereal mixed culture systems. New Zealand Jnl Crop Hort Sci 40, 161-186. Pelissier, F. Improved germination bioassays for allelopathy research. Acta Physiol Plant 2013 35, 23-30.

Reinhardt, C.F., Meissner, R. and Labuschagne, N. (1994). Allelopathic interaction of Chenopodium album L. and certain crop species. Sth Afr Jnl Plant Soil 11, 45-49. 
Santos, S., Moraes, M.L.L. and Rezende, M.O.O. (2010). Allelopathic potential and systematic evaluation of secondary compounds in extracts from roots of Canavalia ensiformis by capillary electrophoresis. Jnl Enviro Sci Health, Part B 45, 325-329.

Shekoofa, A., Safikhan, S., Raper, T. and Butler, S.A. (2020). Allelopathic impacts of cover crop species and termination timing on cotton germination and seedling growth. Agron 10, 638. DOI: 10.3390/agronomy 10050638

Siyar, S., Majeed, A., Muhammad, Z., Ali, H. and Inayat, N. (2019). Allelopathic effect of aqueous extracts of three weed species on the growth and leaf chlorophyll content of bread wheat. Acta Ecol Sinica 39, 63-68.

Smith, M.W., Wolf, M.E., Cheary, B.S. and Carroll, B.L. (2001). Allelopathy of bermudagrass, tall fescue, redroot pigweed, and cutleaf evening primrose on pecan. HortSci 36, 1047-1048.

Sturm, D.J., Peteinatos, G. and Gerhards, R. (2018). Contribution of allelopathic effects to the overall weed suppression by different cover crops. Weed Res DOI: I0.IIII/wre.12316.

Valenzuela, L., Zou, L., Santanen, A. and Mäkelä, P.S.A. (2018). Evaluation of the bioactivity of water extracts from dicotyledonous crops on model weeds and pathogens. Jnl Agric Food Dev 4, $17-22$.

Viard-Crétat, F., Gallet, C., Lefebvre, M. and Lavorel, S. (2009). A leachate a day keeps the seedlings away: mowing and the inhibitory effects of Festuca paniculata in subalpine grasslands. Ann Bot 103, 1271-1278.

Weston, L.A. (2005). History and current trends in the use of allelopathy for weed management. Proceedings $4^{\text {th }}$ world congress allelopathy. Wagga-Wagga, Australia.

Weston, L.A., Alsaadawi, I.S. and Baerson, S.R. (2013). Sorghum allelopathy - from ecosystem to molecule. Jnl Chem Ecol 39, 142-153.

Wu, H., Pratley, J. Lemerle, D., Haig, T. and An, M. (2001). Screening methods for the evaluation of crop allelopathic potential. Botanical Reviews 67, 403-415.

Wynne, K., Adams, C., Neely, C., DeLaune, P., Kimura, E. and Thapa, S. (2019). Canola emergence and early growth were not affected by allelopathic properties of wheat residue. Agrosyst, Geosci Environ 2,1-7. DOI: 10.2134/age2018.11.0058

Zubair, H.M., Pratley, J.E., Sandral, G.A. and Humphries, A. (2017). Allelopathic interference of alfalfa (Medicago sativa L.) genotypes to annual ryegrass (Lolium rigidum). Jnl Plant Res 130, 647-658. DOI: 10.1007/s10265-017-0921-9 